\title{
SOME FINE-TUNING FOR DOMINANT DIAGONAL MATRICES
}

\author{
Carl P. SIMON \\ University of Michigan, Ann Arbor, MI 48109, USA
}

Received 19 August 1988

Accepted 26 October 1988

Given a linear system $\boldsymbol{A x}=\boldsymbol{b}$, where $\boldsymbol{A}$ is a dominant diagonal matrix with positive diagonals and non-positive off-diagonals, but $b$ has both positive and negative components, necessary and sufficient conditions on $b_{j}$ are derived to guarantee that $x_{j}$ is positive.

Dominant diagonal matrices with positive diagonals and non-positive off-diagonals, that is, matrices $A=\left[\left(a_{i j}\right)\right]$ with the property that

$a_{j j}>0, \quad a_{i j} \leq 0(i \neq j)$, and $\sum_{h=1}^{n} a_{h j}>0$ for all $i, j$

play an important role in economic theory because of their two special properties. First, all their eigenvalues have positive real part, a fact which plays a key role in the stability analysis of general equilibria and in the study of gross substitutability. Second, the inverse of such a matrix is a non-negative matrix, so that whenever the vector $\boldsymbol{b}$ has only positive entries, the solution $\boldsymbol{x}$ of the system

$A \boldsymbol{x}=\boldsymbol{b}$

has all positive entries too. This fact is the central ingredient in Leontief's input-output analysis of economic systems and in the Stolper-Samuelson study of world goods prices. See Takayama (1974) and Berman and Plemmons (1979), for example, for detailed discussions of the theory and applications of matrices which satisfy (1).

For simplicity of notation, we will call such matrices ddm-matrices, because they are closely related to M-matrices and to Metzler matrices. They are precisely the dominant diagonal matrices which arise in the above economic models. They also arise naturally in finite difference methods for partial differential equations, in the linear complementarity problem in operations research and in the study of Markov processes in probability and statistics.

In this note, we do some fine-tuning on the second property of ddm-matrices: the positivity of the solution $\boldsymbol{x}$ of (2) when every component of $\boldsymbol{b}$ is positive. In particular, we answer the question: what can one say about $x_{j}$ if $b_{j}>0$ but some other $b_{i}$ 's are negative? This question is especially interesting 
when one is performing comparative statics with the implicit solutions $\left(y_{1}, \ldots, y_{n}\right)$ of a parametrized system of $n$ smooth non-linear equations

$F_{i}\left(y_{1}, \ldots, y_{n} ; z\right)=0, \quad i=1, \ldots, n$.

Differentiating these equations, one is led to a linear system (2) where

$a_{i j}=\mathrm{\partial} F_{i} / \partial_{y j} \quad$ and $\quad x_{j}=\mathrm{d} y_{j} / \mathrm{d} z$

If the resulting $A$ satisfies (1), as it does in Jacquez et al. (1988), then one wants to know the sign of $\mathrm{d} y_{j} / \mathrm{d} z$, even though the right-hand side of (2) may have cntries with both signs.

The following theorem provides necessary and sufficient conditions for concluding that a given component $x_{j}$ of the solution $x$ of (2) is positive.

Theorem 1. Let $\boldsymbol{b}$ be a fixed n-vector. Suppose that $b_{j}>0$ and that

$b_{j}+\sum_{i \in S} b_{i}>0$, where $S \equiv\left\{i=1, \ldots, n: b_{i}<0\right\}$

(a) Then, for any matrix $A$ satisfying (1), the $j$-th component $x_{j}$ of the solution of $A \boldsymbol{x}=\boldsymbol{b}$ is positive.

(b) If (3) is violated, then there exist ddm-matrices $A_{1}$ and $A_{2}$ such that the $j$-th component $x_{j}$ of the solution of $A_{1} x=b$ is positive and the $j$-th component $x_{j}$ of the solution of $A_{2} x=b$ is negative

Remark 1. Condition (3) is easily seen to be equivalent to

$b_{j}+\sum_{i \in T} b_{i}>0, \quad$ for all subsets $T$ of $\{1, \ldots, j-1, j+1, \ldots, n\}$.

This is the condition we will use in the proof of the Theorem 1 and that was used in applying this theorem in Jacquez et al. (1988).

Remark 2. If $x^{*}$ is the solution of $A \boldsymbol{x}=\boldsymbol{b}$, then $-\boldsymbol{x}^{*}$ is the solution of $A \boldsymbol{x}=-\boldsymbol{b}$. It follows that if, in the above notation, $b_{j}<0$ and $b_{j}+\sum_{i}\left\{b_{i}: b_{i}>0\right\}<0$, then $x_{j}<0$.

Remark 3. McKenzie (1959) generalized the classical definition of dominant diagonal matrices to include matrices $A$ for which the product $D A$ satisfies the classical definition for some positive diagonal matrix $D$. If we apply this generalization to (1), we would replace ' $\sum_{h=1}^{n} a_{h j}>0$ for all $j$ ' in (1) by ' $\sum_{h=1}^{n} d_{h} a_{h j}>0$ for all $j$ for some choice of positive numbers $d_{1}, \ldots, d_{n}$ '. For such a matrix $A$, one modifies conditions (3) and (4) simply by multiplying each $b_{h}$ in these conditions by the corresponding $d_{h}$.

Proof of Theorem 1. The proof follows from solving (2) by Gaussian elimination, noting that the square submatrices that result after each pivot are still dominant-diagonal, and keeping track of the 
right-hand sides (RHSs) through this process. Without loss of generality, we will take $j$ to be $n$. To keep better track of the signs and sizes of the entries of the ddm-matrix $A$, we write it as

$A=\left(\begin{array}{rrcr}a_{11} & -a_{12} & \cdots & -a_{1 n} \\ -a_{21} & a_{22} & \cdots & -a_{2 n} \\ \vdots & \vdots & \ddots & \vdots \\ -a_{n 1} & -a_{n 2} & \cdots & a_{n n}\end{array}\right)$, where each $a_{i j} \geq 0 \quad$ and $0 \leq \sum_{n \neq j} a_{h j}<a_{j j}$

for all $j$. Start with the augmented matrix $[A \mid b]$ for system (2). Add $a_{j 1} / a_{11}$ times row one to row $j$ for all $j>1$. The result is the new augmented matrix:

$\left(\begin{array}{cccc:c}a_{11} & -a_{12} & \ldots & -a_{1 n} & b_{1} \\ 0 & a_{22}-\frac{a_{21}}{a_{11}} a_{12} & \ldots & -a_{2 n}-\frac{a_{21}}{a_{11}} a_{1 n} & b_{2}+\frac{a_{21}}{a_{11}} b_{1} \\ \vdots & \vdots & \ddots & \vdots & \vdots \\ 0 & -a_{n 2}-\frac{a_{n 1}}{a_{11}} a_{12} & \ldots & a_{n n}-\frac{a_{n 1}}{a_{11}} a_{1 n} & b_{n}+\frac{a_{n 1}}{a_{11}} b_{1}\end{array}\right) \equiv\left(\begin{array}{cc:c}a_{11} & * & b_{1} \\ \mathbf{0} & \hat{A} & \hat{\boldsymbol{b}}\end{array}\right)$.

The $(n-1) \times(n-1)$ matrix $\hat{A}$ is still dominant diagonal, since its off-diagonal entries are still non-positive and the sum of the entrics in its $\left(\begin{array}{ll}j & 1\end{array}\right)$ th column is

$$
\begin{aligned}
& \left(a_{j j}-\frac{a_{j 1}}{a_{11}} a_{1 j}\right)+\sum_{h \neq 1, j}\left(-a_{h j}-\frac{a_{h 1}}{a_{11}} a_{1 j}\right) \\
& \quad=a_{j j}-\left(\sum_{h \neq 1, j} a_{h j}\right)-a_{1 j} \frac{a_{21}+\cdots+a_{n 1}}{a_{11}}>a_{j j}-\sum_{h \neq 1, j} a_{h j}-a_{1 j}>0 \quad \text { by (5) twice. }
\end{aligned}
$$

The new RHS $\hat{\boldsymbol{b}}$ still satisfies (4): for, $\hat{b}_{n-1}=b_{n}+\left(a_{n 1} / a_{11}\right) b_{1}$ is still positive, since either $b_{1}>0$ or $b_{n}+b_{1}>0$ and $a_{n 1} / a_{11}<1$. In addition, for any subset $I$ of $\{1, \ldots, n-1\}$

$$
\begin{gathered}
\hat{b}_{n-1}+\sum_{i \in I} \hat{b}_{i-1}=b_{n}+\frac{a_{n 1}}{a_{11}} b_{1}+\sum_{i \in I}\left(b_{i}+\frac{a_{i 1}}{a_{11}} b_{1}\right)=b_{n}+\sum_{i \in I} b_{i}+b_{1} \sum_{n, I} \frac{a_{i 1}}{a_{11}} \\
\left\{\begin{array}{lll}
>b_{n}+\sum_{I} b_{i} & \text { if } b_{1}>0, & \text { since } \quad \sum_{i} \frac{a_{i 1}}{a_{11}}>0, \\
>b_{1}+\sum_{1, I} b_{i} & \text { if } b_{1} \leq 0, & \text { since } \quad \sum_{i} \frac{a_{i 1}}{a_{11}}<1,
\end{array}\right.
\end{gathered}
$$$$
>0 \text { by (4). }
$$

The $(n-1) \times(n-1)$ system $\hat{A} \boldsymbol{x}=\hat{\boldsymbol{b}}$ satisfies the hypotheses of Theorem 1 . Continue applying Gaussian elimination; at each stage the hypotheses of the theorem are satisfied. At the last step, the system reduces to the simple equation

$a^{*} x_{n}=b^{*}$, where $a^{*}>0$ and $b^{*}>0$. 
It follows that $x_{n}>0$.

To prove part (b) of Theorem 1, we assume without loss of generality that $b_{1}>0$ and $b_{1}+b_{2}$ $+\cdots+b_{k}<0$ for some $k$. For the conclusion of part (b), take $A_{1}$ to be the identity matrix and take $A_{2}$ to be the ddm-matrix

$\left(\begin{array}{ccccc:ccc}(1+\varepsilon)^{-1} & -(1+\varepsilon)^{-r} & -(1+\epsilon)^{-r} & \ldots & -(1+\varepsilon)^{-r} & 0 & \ldots & 0 \\ 0 & (1+\epsilon)^{-r+1} & 0 & \ldots & 0 & 0 & \ldots & 0 \\ 0 & 0 & (1+\varepsilon)^{-r+1} & \ldots & 0 & 0 & \ldots & 0 \\ \vdots & \vdots & \vdots & \ddots & \vdots & \vdots & \ddots & \vdots \\ 0 & 0 & 0 & \ldots & (1+\varepsilon)^{-r+1} & 0 & \ldots & 0 \\ \hdashline 0 & 0 & 0 & \ldots & 0 & 1 & \ldots & 0 \\ \vdots & \vdots & \vdots & \ddots & \vdots & \vdots & \ddots & \vdots \\ 0 & 0 & 0 & \ldots & 0 & 0 & \ldots & 1\end{array}\right)$

where the top-left block is $k \times k$, the bottom-right block is the $(n-k) \times(n-k)$ identity matrix, and the other two blocks are zero matrices. Choose $\varepsilon$ small enough so that $(1+\varepsilon) h_{1}+b_{2}+\cdots+b_{k}<0$. (If you want $A_{2}$ to be both row- and column-diagonal dominant, choose $r$ big enough so that the first row of $A_{2}$ has a positive sum.) Let $c$ be the row $n$-vector,

$c=(1+\varepsilon, 1, \ldots, 1,0, \ldots, 0)$,

whose last $n-k$ entries are all zeros. Since $c A_{2}=(1,0,0, \ldots, 0), c$ is the first row of $A_{2}^{-1}$. The solution of (2) is $\boldsymbol{x}=A_{2}^{-1} \boldsymbol{b}$ and

$x_{1}=\boldsymbol{c} \cdot \boldsymbol{b}=(1+\varepsilon) b_{1}+b_{2}+\cdots+b_{k}<0$.

An advantage of this approach to dominant diagonal malrices is that it is easy to recover the classical properties of ddm-matrices from the statement and proof of Theorem 1.

Theorem 2. Let $A$ be a matrix which satisfies (1). Then,
(a) $\operatorname{det} A>0$;
(b) $A$ is non-singular;
(c) all eigenvalues of $A$ have a positive real part;
(d) if $\boldsymbol{b}>\mathbf{0}$, then the solution $\boldsymbol{x}$ of $A \boldsymbol{x}=\boldsymbol{b}$ has all components positive;
(e) if $\boldsymbol{b} \geq \mathbf{0}$, then the solution $\boldsymbol{x}$ of $A \boldsymbol{x}=\boldsymbol{b}$ has all components non-negative;
(f) all entries of $A^{-1}$ are non-negative.

Pronf. The proof of Theorem 1 clearly showed that all the pivots of $A$ are positive. Since the determinant is the product of the pivots, $\operatorname{det} A$ is positive and $A$ is non-singular. Since every principal submatrix of $A$ satisfies (1), every principal minor of $A$ is positive. Since the coefficient of $x^{k}$ in the characteristic polynomial of $A$ is $(-1)^{k}$ times the sum of the $k$ th order principal minors, these coefficients alternate in sign. By Descartes' Rule of Signs, all the zeros of the characteristic polynomial of $A$, i.e., the eigenvalues of $A$, have positive real part. Statement (d) follows immediately from Theorem 1, since every component of a positive vector satisfies condition (3). Statement (e) 
follows from statement (d) and the continuity of linear maps. Finally, statement (f) follows statement (g) and the fact that the $j$ th column $\boldsymbol{c}_{j}$ of $A^{-1}$ is the solution $\boldsymbol{x}=\boldsymbol{c}_{j}$ of the system $A \boldsymbol{x}=\boldsymbol{e}_{j}$, where $\boldsymbol{e}_{j}$ is the $j$ th canonical basis vector, a non-negative vector.

\section{References}

Berman, A. and R. Plemmons, 1979, Nonnegative matrices in the mathematical sciences (Academic Press, New York).

Jacquez, J., C. Simon, J. Koopman, L. Sattenspiel and T. Perry, 1988, Modeling and analyzing HIV transmission: The effect of contact patterns, Mathematical Biosciences, submitted for publication.

McKenzie, L.W., 1959, Matrices with dominant diagonals and economic theory, in: K. Arrow, S. Karlin and P. Suppes, eds., Mathematical methods in social sciences (Stanford University Press, Stanford, CA).

Takayama, A., 1974, Mathematical economics (Dryden Press, IIinsdale, IL). 